\title{
Критерии рациональности изменения убеждений: непротиворечивость
}

\author{
Н. П. КОЗАЧЕНКО
}

\begin{abstract}
We consider some possible strategies in explicating the rationality criteria within various directions in belief revision. More specifically, we compare the so called AGM-approach (by Alchourron, Gärdenfors and Makinson) with the Paraconsistent Belief Revision advocated recently by Edwin Mares, as to dealing with inconsistent theories and believe bases. We conclude that although it is hardly possible to eliminate the principle of consistency, it still can be weakened by a suitable concept of coherence. It allows to effectively isolate contradictions and to work rationally with inconsistent believe bases.
\end{abstract}

Ключевые слова: ревизия убеждений, паранепротиворечивая ревизия убеждений, теории типа AGM, критерии рациональности, множества убеждений, баэы убеждений

\section{Введение}

Динамика убеждений и знаний находится в поле зрения доксастической и эпистемической логик. Терминологическое разделение в духе фон Вригта хоть и предполагает наличие доксастической логики убеждений и эпистемической логики знаний [17], но не слишком разграничивает их области исследований. Более того, в контексте исследования познавательных процессов, которые приводят к изменению когнитивного состояния субъекта, в центре рассмотрения чаще всего оказывается обобщенная категория убеждения ${ }^{1}$. Истолкованное в широком смысле множество убеждений включает знания и чаще всего, в данном контексте, термины «знание» и «убеждение» используются

\footnotetext{
${ }^{1}$ По мнению В.Н. Костюка, рассмотрение знания в строгом смысле «в общем случае препятствует рассмотрению возможности развития знания, перехода от менее полного к более полному знанию, игнорирует элемент гипотетичности в (научном) знании» [1, с. 131].
} 
как синонимы, если явно не указано различие между ними. В дальнейшем изложении мы также будем придерживаться этой традиции.

Одной из фундаментальных проблем эпистемической логики является поиск адекватного способа представления знаний и убеждений с учетом специфики человеческого познавательного процесса. Не имея возможности абсолютно точно отобразить действительный способ существования убеждений, исследователи вынуждены прибегать к довольно сильным идеализациям. В авангарде колонны эпистемических идеализаций стоит абстрактный познающий субъект, который активно и рационально производит определенные действия над своими убеждениями. Использование такой сильной идеализации тем не менее стало плодотворной почвой для развития большого количества исследований механизмов существования и изменения знаний и убеждений. Эту отрасль формальной эпистемологии в англоязычной литературе называют изменением теорий (theory change) или ревизией убеждений (belief revision).

Основные принципы исследования способов представления и изменения убеждений сформулированы в концепции, которая получила в литературе название AGM (по заглавным буквам фамилий авторов - К.Алчуррона, П. Герденфорса и Д. Макинсона, предложивших этот подход) [2]. В настоящее время разработан целый ряд теорий, позволяющих в той или иной мере описывать процессы изменения знаний, варьируя способ представления убеждений, критерии рациональности убеждений, возможные познавательные действия. Большинство из них используют основные принципы изменения убеждений концепции AGM, ставшей своего рода классикой belief revision. Чаще всего рассматривают три типа возможного изменения теории: pacuирение (expansion) - высказывание просто добавлено к теории, ревизия (revision) - произведен пересмотр теории на предмет предупреждения появления в ней противоречий, а затем добавлено новое высказывание, сокращение (contraction) - высказывание удалено из теории. 


\section{1 Рациональная непротиворечивость}

Чтобы разработать концептуальный аппарат для исследования динамики знаний и убеждений, следует отыскать подходящий способ представления эпистемических элементов - знаний и убеждений субъекта. Возможный или текущий набор знаний и убеждений субъекта называют эпистемическим состоянием (epistemic state) или состоянием убеждений (belief state). В зависимости от способа представления эпистемического состояния, определяют критерии рациональности, которым они должны соответствовать. Это своего рода метауровень, который описывает правила существования и изменения убеждений. Традиционные критерии, принятые в AGM, характерны практически для всех сентенциональных моделей, которые рассматривают эпистемическое состояние субъекта как множество убеждений (belief set) - высказываний. Каждое высказывание может быть убеждением, равно как и любое убеждение может быть представлено в виде высказывания. Исходное множество убеждений замкнуто относительно логического следования. Эпистемический вход (epistemic input) - информация, которая приводит к изменениям исходного состояния убеждений, также представляется как высказывание.

Критерии рациональности AGM для множества убеждений выражаются требованиями:

• минимальности изменений исходной информации,

- приоритетности новой информации,

- непротиворечивости.

Каждый из перечисленных критериев порождает ряд дискуссий относительно его уместности, необходимости и способа реализации. Часто на основе таких дискуссий развивается новое направление в исследовании процессов изменения убеждений, связанное с определенным способом представления эпистемического состояния и построением соответствующей модели.

Действуя в рамках критериев рациональности, следует также стремится к соблюдению принципа категориального соответствия при выполнении познавательных действий. В формулировке П. Герденфорса и Х. Ротта, этот принцип звучит так: 
В результате выполнения какого либо действия, результат должен представлять собой систему, аналогичную исходной и соответственно обладающую такими же свойствами [7].

В рамках различных традиций представления знаний существуют самые разнообразные воплощения этого принципа, но следует отметить, что практически все существующие системы предпочитают работать с убеждениями прежде всего рационального субъекта, оперирующего непротиворечивыми убеждениями. В данном случае, мы говорим о том, что любое изменение множества убеждений должно выдать результат в виде множества убеждений, также замкнутого относительно логического следования, как и исходное.

Следует отметить, что требования рациональности сами по себе естественны и закономерны, но любая их реализация в рамках определенного представления убеждений зачастую требует очень высокого уровня идеализации. Множество убеждений рационального субъекта должно быть непротиворечиво, потому что следующим требованием, исходящим из способа представления убеждений, есть замкнутость множества убеждений относительно логического следования. Если же субъект проигнорировал требование непротиворечивости, то результатом замыкания такого множества убеждений будет множество всех высказываний языка, что приводит к тривиализации самого процесса пересмотра убеждений. Устранение, своевременное обнаружение и предупреждение появления противоречий во множестве убеждений - это своего рода священная обязанность каждого субъекта - носителя убеждений.

Прежде чем приступить к изменению множества убеждений, следует увериться в его непротиворечивости, и так же завершающим этапом каждой доксастической операции должна быть проверка результата на непротиворечивость. Тем не менее, реальный познающий субъект не так уж и нуждается в постоянном контроле противоречий. Во множестве убеждений обычного человека порой мирно сосуществуют несовместимые высказывания, наличие которых не обязательно есть свидетельством нерациональности, но скорее признаком «человечности». Замкнутость относительно логического следования также необязательна для познающего субъекта, - возможно именно поэтому обыч- 
ный, «живой» носитель множества убеждений не приходит в ужас, осознавая перспективу тривиализации своих убеждений. Постепенно, устраняя проявляющиеся противоречия, человек успешно изменяет свое множество убеждений, порой даже не осознавая всех производимых операций.

Допустив подобную необязательность при построении доксастической логики, мы фактически в самом начале потеряем исходные принципы, на которые она опирается. Для абстрактного рационального субъекта, в идеале реализовать действия которого сможет, к примеру, компьютер, необходимо наличие четких требований и такое же четкое их выполнение. Человек, по-видимому, обладает все же ограниченными познавательными способностями, поэтому он делает необходимые умозаключения ad hoc, когда же получает противоречивые следствия, задумывается и устраняет несоответствие. Компьютер, оперируя аналогичным множеством убеждений, обладает бо́льшими вычислительными возможностями. Кроме того, если «человеку свойственно ошибаться», то компьютеру в такой возможности отказывают. Обнаружив противоречие в своих убеждениях, у человека всегда есть возможность исправить ситуацию. У компьютера подобной возможности в аналогичных условиях нет, ведь получив уже противоречивое множество, он не будет делать заключения лишь по надобности, рискуя выдать ошибочный результат, а немедленно выполнит замыкание относительно логического следования и получит множество всех высказываний языка. Полностью отказаться от требования непротиворечивости множества убеждений нельзя. Но также нельзя игнорировать ситуации, в которых существование противоречия в состоянии убеждений вынуждено ${ }^{2}$.

Способов устранения или локализации противоречия во множестве убеждений достаточно много, но все они проистекают из необходимости соблюдения условия непротиворечивости. Тем не менее, некоторые исследователи предлагают способы ослабить или отказаться от этого критерия. Понятно, что просто

\footnotetext{
${ }^{2}$ Например, экспертная система букмекерского клуба, получает информацию о результатах матча из разных источников. Получив противоречивую информацию, система должна локализовать противоречие и ожидать уточнения.
} 
отбросить его нельзя, ведь в таком случае мы теряем возможность использовать множества убеждений как удобный и привычный способ представления эпистемических состояний. Даже ослабление условия непротиворечивости также влечет изменение способа представления состояния убеждений. Чтобы получить возможность более пристально взглянуть на пути «подкопа под стену непротиворечивости» рассмотрим две характерные доксастические модели.

\section{2 Отказ от непротиворечивости: ревизия базы убеждений}

Рассмотрение человеческих способностей оперировать своими убеждениями часто наталкивает на мысль, что представление этих убеждений в виде множества высказываний не вполне адекватно выражают механизмы изменения состояний убеждений человека. Конечно, мы имеем здесь удобную и сильную идеализацию, формально отражающую большинство аспектов изменения убеждений, но порой ее выразительных возможностей оказывается недостаточно, в частности, при рассмотрении условия непротиворечивости. Как бы ни верили мы в человеческую рациональность, но все же приходится признать, что иногда человек халатно относится к своим эпистемическим обязанностям: не следит за непротиворечивостью множества убеждений, не замыкает его относительно логического следования, и даже не осознает всех следствий своих убеждений. А почему, собственно говоря, он должен это делать?

Множество убеждений как способ представления эпистемического состояния субъекта подвергался критике со стороны многих исследователей, среди которых выделяются А. Фурманн [4], С. Ханссон [9], П. Герденфорс [6], Х. Ротт [7], Б. Небел [15] и другие. Чаще всего указывают на ограниченные описательные возможности аппарата теории множеств применительно к убеждениям, что затрудняет возможности описания статуса убеждения, его зависимостей, подтвержденности или ценности. Д. Макинсон также обращает внимание на неудобство оперирования множеством высказываний, практически бесконечным в силу требования логической замкнутости [13].

Подобных аргументов очень много. Действительно, эпистеми- 
ческое состояние субъекта неоднородно. Не все наши убеждения имеют независимый статус, большая их часть следуют из других, более фундаментальных убеждений. В терминах множества убеждений описать такое различие практически невозможно, не имея отличительного признака для базовых и производных убеждений. Кроме того, указывает А. Фурманн, в процессе изменения убеждений, мы никогда не охватываем все множество убеждений, а работаем лишь с его базовой частью [4], изменение которой во многом похоже на операции над множеством убеждений, но имеет и ряд особенностей.

ОПРЕДЕЛЕНИЕ 1. Множество $B_{K}$ называется базой убеждений множества убеждений $K$, если и только если $B_{K}$ - конечное подмножество $K$ и результатом логического замыкания $B_{K}$ есть само множество $K$.

Для базы убеждений необязательно условие замкнутости относительно логического следования и, соответственно, требование непротиворечивости также не упоминается. А значит, база убеждений может содержать противоречивые высказывания. Фактически, любой набор высказываний представляет собой некоторую базу убеждений. Очевидно, что базы убеждений обладают большими выразительными возможностями, нежели множества убеждений. Одному множеству убеждений могут соответствовать разные базы. Фактически, база убеждений представляет собой набор основных, фундаментальных убеждений, на основе которых формируется то или иное множество убеждений.

Изменение базы убеждений осуществляется с помощью тех же трех операций: расширения, сокращения и ревизии. С учетом принципа категориального соответствия, результатом выполнения некоторого действия над базой убеждений также должна быть база убеждений. Таким образом, расширение базы убеждений выглядит несколько проще расширения множества убеждений, где для достижения категориального соответствия необходимо замкнуть результат относительно логического следования. Расширение базы убеждений предполагает лишь механическое добавление нового высказывания ко множеству уже существующих в базе. 
ОПРЕДЕЛЕНИЕ 2. Расширением базы убеждений $B$ посредством высказывания $\alpha$ назовем операцию + , такую что $B+\alpha=$ $B \cup\{\alpha\}$.

В работах Ханссона функция сокращения интерпретирована для баз убеждений, путем введения дополнительного оператора - оператора ядра [10]. Такой оператор на множестве высказываний $B$ выбирает для любого высказывания $\alpha$ минимальное подмножество исходного множества $B$, такое что оно влечет $\alpha$.

ОПРЕДЕЛЕНИЕ 3. Для любого множества высказываний $B$ и любой формулы $\alpha$, назовем оператор $\Perp$ оператором ядра, если и только если для любого множества высказываний $X \in B \Perp \alpha$ выполняются следующие условия:

1. $X \subseteq B$,

2. $X \models \alpha$,

3. для любого $Y$, если $Y \subset X$ то $Y \not \neq \alpha$.

$B \Perp \alpha$ называется центральным $\alpha$-множеством, его элементы $\alpha$-ядрами. Каждая база убеждений порождает ряд $\alpha$-ядер, т.е. минимальных подмножеств базы, которые влекут $\alpha$. Сокращение баз убеждений реализовано Ханссоном через сокращение ядpa (kernel contraction) [10]. Суть этой операции довольно проста. Чтобы сократить базу убеждений посредством высказывания $\alpha$, потребуется:

- выбрать все $\alpha$-ядра базы убеждений;

- удалить наименее предпочтительный элемент каждого $\alpha$ ядра.

В результате выполнения этих действий, полученное множество убеждений уже не будет влечь $\alpha$, так как ни одна его база не будет влечь $\alpha$.

Для выбора наименее предпочтительных элементов ядра, подлежащих удалению, используется функиия усечения (incision function), которая определяет, какой элемент каждого $\alpha$-ядра следует удалить. Функция усечения $\sigma$ в качестве аргумента принимает центральное $\alpha$-множество и производит выбор на объединении всех минимальных подмножеств, влекущих $\alpha$ ( $\alpha$-ядер). При этом $\sigma$ гарантирует, что в каждом $\alpha$-ядре будет выбрано хотя бы одно высказывание, подлежащее удалению. 
ОПРЕДЕЛЕНИЕ 4. Функцией усечения для $B$ назовем такую функцию $\sigma$, которая для любого высказывания $\alpha$ будет удовлетворять следующим условиям:

1. $\sigma(B \Perp \alpha) \subseteq \cup(B \Perp \alpha)$,

2. если $X \neq \varnothing$ и $X \in B \Perp \alpha$, то $X \cap \sigma(B \Perp \alpha) \neq \varnothing$.

Используя функцию усечения $\sigma$, Ханссон определяет сокращение ядра для базы убеждений [10].

ОПРЕДЕЛЕНИЕ 5. Сокращением ядра для базы убеждений $B$ на основе функции усечения $\sigma$, назовем операцию $-{ }_{\sigma}$, такую что $B-_{\sigma}=B \backslash \sigma(B \Perp \alpha)$.

Сокращение ядра базы убеждений удовлетворяет постулатам сокращения AGM, кроме постулата замыкания. Сокращение ядpa, которое бы удовлетворяло всем AGM постулатам сокращения, может быть построено с использованием функи,и мягкого усечения (smooth incision function).

ОПРЕДЕЛЕНИЕ 6. Функцию $\sigma$ усечения на множестве $B$ назовем функцией мягкого усечения, если и только если для любого $A \subseteq B$, если $A \vdash \beta$ и $\beta \in \sigma(B \Perp \alpha)$, то $A \cap \sigma(B \Perp \alpha) \neq \varnothing$.

Соответствующая операчия мягкого сокращения ядра, основанная на функции мягкого усечения, удовлетворяет всем постулатам сокращения AGM; исходя из этого, Ханссон формулирует и доказывает репрезентационную теорему [11].

TEOPEMA 7. Пусть K есть множество убеждений. Оператор - на K будет оператором мягкого сокращения ядра, если и только если - есть оператор сокращения частичного пересечения.

Ревизия базы убеждений может быть представлена с использованием равенства Леви: $K * \alpha=(K-\neg \alpha)+\alpha$, причем в данном случае порядок действий значения не имеет. Требование непротиворечивости для множества убеждений четко определяет порядок осуществления ревизии [7]:

- удаляем предполагаемое противоречие $(\neg \alpha)$;

- добавляем высказывание $\alpha$.

Аналогичный порядок действий можно сохранить и для ревизии базы убеждений, определив ее на основе расширения и 
сокращения базы убеждений, рассмотренных выше. Специфическим для баз убеждений случаем оказывается операция полуревизии (semirevision) базы $B$ посредством убеждения $\alpha$, соответствующая обратному равенству Леви [11]: $K * \alpha=(K+\alpha)-\neg \alpha$.

Иными словами, полуревизия базы убеждений предполагает два следующих шага:

1. Расширение базы убеждений $B$ посредством $\alpha$.

2. Удаление противоречий в $B$, если таковые возникли (консолидаиия базы).

ОПРЕДЕЛЕНИЕ 8. Полуревизия ядра базы убеждений, основанная на функции усечения $\sigma-$ это операция вида $\circledast_{\sigma}$, для которой $B \circledast_{\sigma} \alpha=B \cup\{\alpha\} \backslash \sigma((B \cup\{\alpha\}) \Perp \perp)$.

Такая операция может дать результат, отличный от результата обычной ревизии, т.к. на втором шаге, удаляя возникшие в базе $B$ противоречия, мы не всегда можем исключить возможность удаления убеждения $\alpha$, посредством которого осуществлялся пересмотр базы. Поэтому операция такого пересмотра базы убеждений и названа полуревизией [9]. При этом не следует снижать ее познавательной ценности, ведь в сущности, она более тонко моделирует процесс пересмотра убеждений. Действительно, когда мы в соответствии со стандартной процедурой, предусмотренной равенством Леви, заранее предпринимаем действие по недопущению противоречия, сокращая состояние убеждений посредством $\neg \alpha$, мы автоматически отвергаем возможность недостоверности $\alpha$, т.е. фактически отдаем предпочтение новой информации перед уже имеющейся. В случае же применения операции полуревизии принцип приоритетности новой информации перестает быть обязательным. И действительно, получая новую информацию, которая к тому же противоречит имеющимся убеждениям, мы скорее отнесемся к ней негативно или настороженно, но, во всяком случае, далеко не всегда будем воспринимать ее как безусловно приоритетную. Эта ситуация реализуется операцией полуревизии - мы принимаем $\alpha$ как равноправное убеждение базы и уже после этого начинаем искать несоответствия, при этом в ходе удаления возникших 
противоречий вполне возможна потеря новой информации $\alpha$, которая может оказаться неконкурентоспособной по сравнению с базовыми убеждениями, хотя бы в силу меньшей субъективной значимости.

Операция полуревизии должна удовлетворять следующим постулатам [11]:

1. Непротиворечивость: $\perp \notin C n(B \circledast \alpha)$

2. Включение: $\mathrm{B} \circledast \alpha \subseteq \mathrm{B} \cup\{\alpha\}$

3. Сохранение ядра: Если $\beta \in \mathrm{B} \backslash \mathrm{B} \circledast \alpha$, тогда существует такое $B^{\prime} \subseteq \mathrm{B} \cup\{\alpha\}$, для которого $\perp \notin C n\left(B^{\prime}\right)$ и $\perp \notin C n\left(B^{\prime}\right) \cup\{\beta\}$

4. Предварительность расширения: $(B+\alpha) \circledast \alpha=B \circledast \alpha$

5. Конгруэнтность: Если $\alpha \leftrightarrow \beta$ теорема, то $B \circledast \alpha=B \circledast \beta$

Использование баз убеждений не освобождает нас от необходимости контролировать возникновение противоречий, но предоставляет возможность существенно уточнить представление эпистемического состояния субъекта и построить более адекватную модель изменения убеждений для моделирования процессов, приближенных к действительному механизму изменения убеждений.

\section{3 Ослабление непротиворечивости: паранепротиворечивая ревизия убеждений}

Возможен и другой способ представления эпистемического состояния субъекта посредством некоторой содержателъной структуры, компонентами которой являются два множества. Первое - множество убеждений, которые субъект в данный момент считает истинными, - принимаемое множество убеждений (acceptance set) (Г). В качестве второго компонента содержательной структуры выступает множество отвергаемых убеждений (rejection set) $(\Delta)$. Уместность рассмотрения множества убеждений, в истинности которых субъект в данный момент уверен, не вызывает сомнений. Напротив, придание отвергаемым высказываниям статус отдельного, причем равноправного, множества содержательной структуры, вызывает закономерный 
интерес и требует уточнения [8]. Отвержение убеждения - это познавательное действие, которое в разговорной речи выражается различными степенями отрицания. Хотя исследования сущности отрицания имеют массивные исторические корни, уходящие в глубину веков к истокам логики, исследователи далеко не всегда обращали внимание на существенное различие между отрицанием утверждения и утверждением отрицания (см. T. Парсонс [16]). Акт речевого отрицания $\alpha$ вынуждает нас отказаться от утверждения $\alpha$, но никоим образом не обязывает полностью отбрасывать отрицаемое высказывание, удалять его и старательно забывать как постыдное свидетельство нашего заблуждения. Естественно, существуют и другие эпистемические обязательства субъекта, согласно которым, в частности, если субъект отвергает некоторое убеждение, он не имеет права одновременно утверждать это убеждение. Используя такой способ ограничения, мы получаем компоненты содержательной структуры:

$\Gamma$ - множество принимаемых убеждений,

$\Delta$ - множество отвергаемых убеждений.

Кроме того, упомянутые выше эпистемические обязательства субъекта не только закономерны и интуитивно понятны, но также могут быть использованы в качестве искомого более слабого заменителя непротиворечивости множества убеждений. Используя представление эпистемического состояния субъекта в терминах содержательной структуры, требование непротиворечивости может быть заменено более слабым - согласованностъю. В данном случае согласованность выполняет функции разделителя компонентов содержательной структуры, - множества принимаемых и отвергаемых убеждений должны быть согласованы, т.е. не иметь общих элементов.

ОПРЕДЕЛЕНИЕ 9. Содержательной структурой Con назовем упорядоченную пару множеств $(\Gamma, \Delta)$, где $\Gamma \cap \Delta=\varnothing$.

Используя возможности рассмотренного представления, Эдвин Марес создает стройную и выразительную модель под названием паранепротиворечивая теория ревизии убеждений (да- 
лее $\left.-\mathrm{PBR}^{3}\right)$ [14]. Построение подобной теории продиктовано необходимостью выработки средств для рациональной работы с противоречиями, если они возникают. Используя непротиворечивость как движущую силу изменения убеждений, невозможно представить себе наличие противоречия во множестве убеждений без тривиализации этого множества. Положив в основу PBR паранепротиворечивую, а не классическую логику, можно рассчитывать получить теорию, в рамках которой допустимо оперировать противоречиями, не опасаясь тривиализации. Разумеется, даже использование паранепротиворечивой логики не может полностью застраховать субъекта от появления противоречий, недопустимых в рамках подобной теории. Поэтому в качестве более слабого аналога непротиворечивости выдвигается требование согласованности множеств принимаемых и отвергаемых убеждений.

Стандартное представление изменения убеждений в теориях типа AGM среди основных принципов использует непротиворечивость и минимальность. Реализация этих принципов подразумевает оптимальный выбор, который позволяет свести к минимуму потери при изменении состояния убеждения. AGM демонстрирует здесь определенную осторожность, которая заключается в стремлении оставить как можно больше высказываний нетронутыми, удалив лишь «наихудшие» убеждения, например, наименее укорененные, наименее подтвержденные, наименее предпочитаемые и т.п.

При построении паранепротиворечивой теории, Э. Марес придерживается аналогичных двух принципов: согласованность и минимальностъ. В целях сохранения согласованности содержательной структуры нельзя допускать нарушения эпистемических обязательств субъекта во избежание пересечения множества принимаемых и множества отвергаемых убеждений. Минимальность PBR состоит в удалении лишь наименее значимых для субъекта убеждений, при этом следует учитывать необходимость минимального изменения обоих множеств - принимаемых убеждений $\Gamma$ и отвергаемых убеждений $\Delta$.

В основе PBR лежит релевантная логика системы $\mathbf{R}$, при

\footnotetext{
${ }^{3} \mathbf{P B R}$ - аббревиатура-сокращение названия модели - Paraconsistent Theory Of Belief Revision.
} 
этом Марес указывает, что выбор логики продиктован только его личными предпочтениями и в качестве основы теории может быть использована любая релевантная или полурелевантная логика от $\mathbf{B}$ до $\mathbf{R} \mathbf{M} 3^{4}$. В качестве метаязыка использована классическая логика первого порядка.

Рассмотрим отношение следования и операторы замыкания для каждого множества - компонента содержательной структуры [8].

ОПРЕДЕЛЕНИЕ 10. Отношение следования. $\Gamma \vdash \Delta$ тогда и только тогда, когда существуют такие наборы высказываний $\gamma_{1}, \gamma_{2}, \gamma_{3} \ldots \gamma_{n} \in \Gamma$ и $\delta_{1}, \delta_{2}, \delta_{3} \ldots \delta_{m} \in \Delta$, что $\gamma_{1} \& \gamma_{2} \&$ $\gamma_{3} \& \ldots \& \gamma_{n} \rightarrow \delta_{1} \vee \delta_{2} \vee \delta_{3} \vee \ldots \vee \delta_{m}$

ОПРЕДЕЛЕНИЕ 11. Оператор восходящего замыкания. Пусть $\Phi-$ множество формул, тогда $C n(\Phi)=\alpha: \Phi \vdash \alpha$.

ОПРЕДЕЛЕНИЕ 12. Оператор нисходящего замыкания. Пусть $\Phi-$ множество формул, тогда $D o(\Phi)=\alpha: \alpha \vdash \Phi$.

Определенные таким образом отношение следования и операторы восходящего замыкания $C n$ и нисходящего замыкания $D o$ имеют следующие свойства.

1. $\alpha \in C n(\Phi)$ и $\beta \in C n(\Phi) \Rightarrow \alpha \wedge \beta \in C n(\Phi)$

2. $\alpha \in D o(\Phi)$ и $\beta \in D o(\Phi) \Rightarrow \alpha \vee \beta \in D o(\Phi)$

3. $C n(\Phi)=C n(C n(\Phi))$

4. $D o(\Phi)=D o(D o(\Phi))$

5. $\left(\Phi \vdash \Phi^{\prime} \& \Phi^{\prime} \vdash \Phi^{\prime \prime}\right) \supset \Phi \vdash \Phi^{\prime \prime}$

6. $\forall \Phi, \Phi \nvdash \varnothing$ и $\forall \Phi, \varnothing \nvdash \Phi$

7. $C n(\varnothing)=D o(\varnothing)=\varnothing$

8. $\left(\Phi \subseteq \Phi^{\prime} \& \Phi^{\prime} \vdash \Phi^{\prime \prime}\right) \supset \Phi^{\prime} \vdash \Phi^{\prime \prime}$

9. $\left(\Phi \subseteq \Phi^{\prime} \& \Phi^{\prime \prime} \vdash \Phi\right) \supset \Phi^{\prime \prime} \vdash \Phi^{\prime}$

\footnotetext{
${ }^{4}$ Подробнее специфику $\mathbf{R}$ и подобных систем см. [3].
} 
Следует отметить, что множество принимаемых убеждений $\Gamma=C n(\Gamma)$ и, соответственно, множество отвергаемых убеждений $\Delta=D o(\Delta)$. Доксастические операции расширения, сокращения и ревизии в терминах PBR могут быть определены аналогично принципам построения операций, описанным в AGM [13]. Описание операции расширения для обоих множеств довольно очевидно.

ОПРЕДЕЛЕНИЕ 13. (Расширение РВR)

Операция расширения для множества принимаемых убеждений: $\Gamma+\alpha=C n(\Gamma \cup\{\alpha\})$.

Операция расширения для множества отвергаемых убеждений: $\Delta \oplus \alpha=D o(\Delta \cup\{\alpha\})$.

Определение операции сокращения всегда более трудоемко и часто требует введения дополнительных понятий. Построение сокращения в терминах PBR Э. Марес осуществляет аналогично сокращению частичного пересечения AGM [2]. Прежде чем приступить к рассмотрению специфики сокращения принимаемого и отвергаемого множеств, следует определить критерии успешности сокращения PBR. Для этого нам понадобятся так называемые множества «остатков» (reminders) относительно некоторого высказывания [8]. Обозначим через $\Gamma \perp \alpha$ множество остатков относительно высказывания $\alpha$ принимаемых убеждений. Аналогично для множества остатков относительно высказывания $\alpha$ отвергаемых убеждений используем запись $\Delta T \alpha$.

ОПРЕДЕЛЕНИЕ 14. Множество $\Gamma^{\prime}$ назовем принимаемым множеством остатков Г по $\alpha$, если и только если для него выполняются следующие условия:

1. $\Gamma^{\prime}$ - множество принимаемых убеждений,

2. $\Gamma^{\prime} \subseteq \Gamma$,

3. $\alpha \notin \Gamma^{\prime}$,

4. $\forall \Gamma^{\prime \prime}:\left(\Gamma^{\prime} \subset \Gamma^{\prime \prime} \subseteq \Gamma\right) \supset \Gamma^{\prime \prime} \vdash \alpha$.

Фактически $\Gamma^{\prime \prime}$ представляет собой множество, содержащее максимальные элементы семейства подмножеств $\Gamma$, которые не влекут $\alpha$.

ОПРЕДЕЛЕНИЕ 15. Множество $\Delta^{\prime}$ назовем отвергаемым множеством остатков $\Delta$ по $\alpha$, если и только если для него выполняются следующие условия: 
1. $\Delta^{\prime}$ - множество принимаемых убеждений,

2. $\Delta^{\prime} \subseteq \Delta$,

3. $\alpha \notin \Delta^{\prime}$,

4. $\forall \Delta^{\prime \prime}:\left(\Delta^{\prime} \subset \Delta^{\prime \prime} \subseteq \Delta\right) \supset \alpha \vdash \Delta^{\prime \prime}$.

Успешность сокращения PBR может быть показана путем доказательства двух лемм о непустоте множеств $\Gamma \perp \alpha$ принимаемых остатков и $\Delta T \alpha$ отвергаемых остатков [14].

Кроме того, в рамках PBR определена пара функций предпочтения:

$$
\begin{aligned}
& \sigma(\Gamma, \alpha) \subseteq \Gamma \perp \alpha \quad(\Gamma \perp \alpha-\text { непусто }), \\
& \tau(\Delta, \alpha) \subseteq \Delta \top \alpha \quad(\Delta \top \alpha-\text { непусто }) .
\end{aligned}
$$

Каждая функция предпочтения выбирает множество высказываний, которые субъект более всего склонен изъять из множества принимаемых или отвергаемых убеждений. Значение функции может зависеть от личных предпочтений субъекта или же от условий выбора.

ОПРЕДЕЛЕНИЕ 16. (Сокращение РВR)

Операция сокращения для множества принимаемых убеждений: $\Gamma-\alpha=\cap \sigma(\Gamma \perp \alpha)$.

Операция сокращения для множества отвергаемых убеждений: $\Delta \ominus \alpha=\cap \tau(\Delta \top \alpha)$.

Определенная таким образом операция сокращения подчиняется требованию категориального соответствия: в результате сокращения множества принимаемых убеждений, получаем принимаемое множество и, соответственно, после сокращения отвергаемого множества, результат также будет представлять собой множество отвергаемых убеждений. Используя определение операций сокращения $\mathrm{PBR}$, функций предпочтения и свойство минимальности множеств остатков, несложно доказать две леммы об успешности каждого вида сокращения. Соответственно, результат $\Gamma-\alpha$ всегда определен и $\alpha \notin \Gamma-\alpha$, равно как всегда определен и успешен результат $\Delta \ominus \alpha, \alpha \notin \Delta \ominus \alpha$.

Для последующего определения операции ревизии убеждений, дополнительно рассмотрим так называемое множественное сокращение. Фурман и Ханссон [5] выделяют два вида множественного сокращения: последовательное и пакетное, в рамках данной теории, автор использует именно пакетное сокраще- 
ние убеждений. Остатки компонентов содержательной структуры по множеству $\Sigma$ могут быть определены аналогично остаткам по некоторому высказыванию. Пакетные остатки $\Gamma \perp \Sigma$ множества $Г$ принимаемых убеждений по множеству $\Sigma$ и $\Delta T \Sigma$ множества отвергаемых убеждений $\Delta$ по множеству $\Sigma$ имеют свойства, полностью аналогичные соответствующим остаткам по единичному высказыванию. Успешность пакетного сокращения обосновывается путем доказательства соответственных лемм о непустоте множеств принимаемых остатков $\Gamma \perp \Sigma$ и отвергаемых остатков $\Delta T \Sigma$ и определением пары функций предпочтения $\sigma^{\prime}(\Gamma, \Sigma) \subseteq \Gamma \perp \Sigma$ и $\tau^{\prime}(\Delta, \Sigma) \subseteq \Delta \top \Sigma$.

ОПРЕДЕЛЕНИЕ 17. (Пакетное сокращение РВR)

Операция пакетного сокращения для множества принимаемых убеждений: $\Gamma-{ }^{\forall} \Sigma=\cap \sigma^{\prime}(\Gamma \perp \Sigma)$.

Операция пакетного сокращения для множества отвергаемых убеждений: $\Delta \Theta^{\forall} \Sigma=\cap \tau^{\prime}(\Delta T \Sigma)$.

Доказательство успешности пакетных сокращений PBR аналогично доказательству соответствующих лемм для единичного сокращения PBR.

Вооружившись определениями расширения и сокращения, можно приступать к рассмотрению комплексного механизма содержательной ревизии. Содержательная ревизия PBR может быть реализована двумя путями, соответственно с помощью

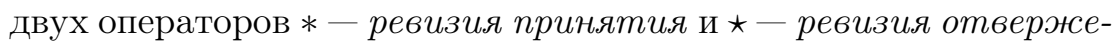
ния. Пусть определена содержательная структура $C$ on $=(\Gamma, \Delta)$. Необходимо ввести в Con высказывание $\alpha$. Э. Марес предлагает следующий алгоритм:

1. Если полученная в результате структура $(\Gamma+\alpha, \Delta \ominus \alpha)$ согласована, то собственно она и будет результатом ревизии.

2. Если же полученная структура несогласована, то действуем по такому алгоритму:

- подвергаем сокращению оба компонента структуры до получения когерентной пары;

- получаем $\Gamma^{\prime \prime} \subseteq \Gamma$ и $\Delta^{\prime \prime} \subseteq \Delta$, как компоненты согласованной структуры $\left(\Gamma^{\prime \prime}+\alpha, \Delta^{\prime \prime}\right)$; 
- определяем $\Gamma^{\prime}=\cap\left(\Gamma^{\prime \prime}+\alpha\right), \Delta^{\prime}=\cap \Delta^{\prime \prime}$;

- на основе компонентов $\Gamma^{\prime}$ и $\Delta^{\prime}$ конструируем содержательную структуру $C o n * \alpha=\left(\Gamma^{\prime}+\alpha, \Delta^{\prime}\right)$, которая и будет результатом содержательной ревизии принятия * [14].

Построение $C$ on $\star \alpha$ аналогично. В случае несогласованности компонентов структуры $(\Gamma-\alpha, \Delta \oplus \alpha)$, следует выполнить действия по согласованию структуры и рассмотреть множества $\Gamma^{\prime \prime} \subseteq \Gamma$ и $\Delta^{\prime \prime} \subseteq \Delta$, при которых структура $\left(\Gamma^{\prime \prime}-\alpha, \Delta^{\prime \prime} \oplus \alpha\right)$ согласована. Затем сконструировать результирующую структуру ревизии отвержения $C o n \star \alpha=\left(\Gamma^{\prime}, \Delta^{\prime} \oplus \alpha\right)$ на основе компонентов $\Gamma^{\prime}=\cap \Gamma^{\prime \prime}, \Delta^{\prime}=\cap\left(\Delta^{\prime \prime} \oplus \alpha\right)$.

Далее Марес предлагает определить функцию выбора $\pi$, которой предстоит выбирать множества убеждений, подлежащие удалению.

Предположим, что структура $(\Gamma+\alpha, \Delta \ominus \alpha)$ несогласованна. Значит, существует по крайней мере одно высказывание $\gamma \in \Gamma$ и хотя бы одно высказывание $\delta \in(\Delta \ominus \alpha)$ такие, что $\alpha \wedge \gamma \vdash \delta$.

Также известно, что все $\gamma$ и $\delta$ в силу замкнутости $\Gamma+\alpha$ относительно $\wedge$ и замкнутости $\Delta \ominus \alpha$ относительно $\vee$, обладают следующими свойствами: $\gamma \nvdash \delta$ и $\alpha \nvdash \delta$.

Кроме того, $\alpha \nvdash \Delta \ominus \alpha$ в силу успешности сокращения.

Рассмотрев множество всех пар $(\gamma, \delta)$, обладающих указанным свойством, можно построить множество множеств, каждое из которых содержит только один член каждой пары и никаких других формул. Обозначим такое множество через $\Pi(\Gamma+\alpha, \Delta \ominus$ $\alpha)$. Если пар $(\gamma, \delta)$, таких что $\alpha \wedge \gamma \vdash \delta$ нет, то $\Pi(\Gamma+\alpha, \Delta \ominus \alpha)=$ $\{\varnothing\}$. Если рассматриваемое множество непусто, то очевидно, что выбрав любой элемент множества $\Pi(\Gamma+\alpha, \Delta \ominus \alpha)$ и сократив с его помощью $\Gamma+\alpha$ и $\Delta \ominus \alpha$, получим согласованную содержательную структуру.

Выбор элемента зависит опять же от личных предпочтений субъекта. Обычно субъект предпочитает выбирать среди наихудших множеств, наименее приемлемых, наименее ценных или значимых убеждений. Обозначим такой выбор как $\pi(\Gamma+\alpha, \Delta \ominus$ $\alpha)$, он существует всегда, если $\Pi(\Gamma+\alpha, \Delta \ominus \alpha)$ непусто. Таким образом, в процессе содержательной ревизии нам предстоит вы- 
полнить сокращение посредством объединения $\pi(\Gamma+\alpha, \Delta \ominus \alpha)$, что возможно с помощью пакетного сокращения.

ОПРЕДЕЛЕНИЕ 18. (Содержательная ревизия принятия)

Con $* \alpha=\left(\Gamma^{\prime}, \Delta^{\prime}\right)$, где $\Gamma^{\prime}=\left(\left(\Gamma-{ }^{\forall} \cap \pi(\Gamma+\alpha, \Delta \ominus \alpha)\right)+\alpha\right)$.

Производя сокращение обоих множеств, и принимаемых, и отвергаемых убеждений посредством объединения наихудших множеств проблемных формул, в результате получаем согласованную пару.

Для оператора $\star$ содержательной ревизии отвержения Э. Марес рассматривает аналогичную функцию выбора $\chi$. Рассмотрению подлежит множество $X(\Gamma-\alpha, \Delta \oplus \alpha)$ множеств формул, состоящих из одного члена каждой пары $(\gamma, \delta)$ и не содержащих при этом никаких других формул. Функция $\chi$ на множестве $X(\Gamma-\alpha, \Delta \oplus \alpha)$ выберет подмножество наихудших проблемных формул, т.е. те множества, которые субъект предпочтет удалить.

$$
\begin{aligned}
& \chi(\Gamma-\alpha, \Delta \oplus \alpha) \subseteq X(\Gamma-\alpha, \Delta \oplus \alpha), \\
& \chi(\Gamma-\alpha, \Delta \oplus \alpha) \text { определена, если } X(\Gamma-\alpha, \Delta \oplus \alpha) \text { непусто. }
\end{aligned}
$$

ОПРЕДЕЛЕНИЕ 19. (Содержательная ревизия отвержения)

Con $\star \alpha=\left(\Gamma^{\prime \prime}, \Delta^{\prime \prime}\right)$, где $\Gamma^{\prime \prime}=\left(\left(\Gamma-{ }^{\forall} \cap \chi(\Gamma-\alpha, \Delta \oplus \alpha)\right)+\alpha\right)$.

Оба определения содержательной ревизии $\mathrm{PBR},-$ принятия * и отвержения $\star,-$ представляют собой варианты равенства Леви. В данном случае, в отличие от ревизии базы убеждений, порядок операций строго определен [14]. Хотя метод достижения согласования принимаемых и отвергаемых множеств не исключает возможность определения оператора полуревизии [11]. Категориальное соответствие содержательных ревизий PBR может быть показано путем несложного доказательства соответствующей теоремы, но более интересными оказываются формальные свойства построенных операторов.

Оба оператора представляют собой типы ревизии, удовлетворяющие постулатам AGM для ревизии [7].

1. Замыкание: $\Gamma=C n(\Gamma)$ и $\Delta=D o(\Delta)$

2. Успешность: $\alpha \in \Gamma^{\prime}$

3. Включение: $\Gamma^{\prime} \subseteq \Gamma+\alpha$ и $\Delta^{\prime} \subseteq \Delta$ 
4. Конгруэнтность: Если $\alpha \leftrightarrow \beta$ теорема $\mathrm{R}$, то $C$ on $* \alpha=$ Con $* \beta$

Отброшенные при построении модели постулаты, соответствующие AGM принципам непротиворечия и минимальности для PBR ревизий, не выполняются.

\section{4 Согласованность vs обоснованность}

Рассмотренные доксастические модели относят к отдельным направлениям развития эпистемических теорий, опирающихся либо на принцип согласованности (coherence), или же на принцип обоснованности (foundations). Основное отличие этих подходов заключается в способе представления эпистемического состояния, его логической структуры.

В рамках подхода, использующего критерий согласованности, убеждения представлены в качестве однородных элементов и подразумевается, что механизм выбора удаляемых убеждений будет осуществлен извне, - его определит либо сама доксастическая операция, либо субъект. K теориям согласованности относятся множества убеждений AGM и рассмотренная выше паранепротеворечивая модель PBR. Такие теории часто упрекают в недостаточной ясности и адекватности представления механизма изменения убеждений, чрезмерной идеализации, статичности и отсутствии четкого алгоритма. Некоторые исследователи называют такие теории «постулатными» и указывают, что рассмотрение свойств убеждений опережает создание ясной онтологии убеждений. В то же время, увеличивающееся количество репрезентационных теорем говорит о том, что в рамках таких моделей фундаментально описаны основные операции изменения, постулаты и свойства познавательных действий, область применения которых подлежит дальнейшему расширению.

Теории, предполагающие обоснованность убеждений, вводят механизм различения статусов убеждений в сам способ их представления (например, оправдательную структуру), таким образом пытаясь изнутри контролировать механизм изъятия убеждений. В качестве примера такой модели было рассмотрено разделение высказываний на эксплицитные и имплицитные через введение баз убеждений. Модели, использующие эпистемическое укоренение, ранжирование и упорядочивание убеждений, 
также относятся к теориям обоснованности. Большинство из них используют основные постулаты сокращения и ревизии AGM, соответствие которым устанавливается путем доказательства репрезентационных теорем. Подобные теории актуальны, так как предоставляют неплохие вычислительные, в частности, алгоритмические возможности, но они также имеют ряд спорных вопросов.

При выборе критериев рациональности естественно опереться прежде всего на здравый смысл и ожидаемый результат. Все же, несмотря на интуитивную понятность и даже необходимость, критерии рациональности эпистемических теорий не являются незыблемыми, даже если цели их построения весьма похожи. Более того, очевидно, что они находятся во взаимозависимости со способом представления убеждений и другими компонентами эпистемической теории. Изменение критерия рациональности влечет за собой иной способ представления состояния убеждений, возможно более удачный, но в то же время, способ представления убеждений диктует особенности воплощения критериев рациональности, их уточнение или даже, в некотором роде, отказ от традиционно принятых.

\section{Литература}

[1] Костюк В.Н. Элементы модальной логики. Киев, 1978.

[2] Alchourron C.E., Makinson D. The logic of theory change: Contraction functions and their associated revision functions // Theoria. 1982. Vol. 48. P. 14-37.

[3] Anderson A.R., Belnap N.D., Dunn J.M. Entailment: Logic of Relevance and Necessity. Volume II. Princeton University Press. 1992.

[4] Fuhrmann A. Theory contraction through base contraction // Journal of Philosophical Logic. 1991. Vol. 20. P. 175-203.

[5] Fuhrmann A., Hansson S.O. A survey of multiple contractions // Journal of Logic, Language and Information. 1994. Vol. 3. P. 39-76.

[6] Gärdenfors P. The dynamics of belief systems: Foundations versus coherence theories // Reveu Internationale de Philosophie. 1990. Vol. 44. P. 24-46.

[7] Gärdenfors P., Rott H. Belief revision. In Handbook of Logic in Artificial Intelligence and Logic Programming. Volume IV. Chapter 4.2. 1995.

[8] Gomolinska A. On the logic of acceptance and rejection // Studia Logica. 1998. Vol. 60. P. 233-251.

[9] Hansson S.O. Belief Base Dynamics, Uppsala: Acta Universitatis Upsaliensis. 1991.

[10] Hansson S.O. Kernel contraction // Journal of Symbolic Logic. 1994. Vol. 59. P. 845-859.

[11] Hansson S.O. Semi-revision // Journal of Applied Non Classical Logic. 1997. Vol. 7. № 1-2. P. 151-175.

[12] Hintikka J. Knowledge and Belief: an Introduction to the Logic of the Two Notions. Ithaca, N. Y.: Cornell University Press, 1962. 
[13] Makinson D. How to give it up: A survey of some formal aspects of the logic of theory change // Synthese. 1985. Vol. 62. P. 347-363.

[14] Mares E.D. A paraconsistent theory of belief revision // Erkenntnis. 2002. Vol. 56. P. 229-246.

[15] Nebel B. Reasoning and Revision in Hybrid Representation Systems. Lecture Notes in Computer Science. 1990. Vol. 422. Berlin: Springer, 1990.

[16] Parsons T. Assertion, denial and the liar paradox // Journal of Philosophical Logic. 1984. Vol. 13. P. 137-152.

[17] Von Wright G.H. An Essay in Modal Logic. Amsterdam: North-Holland, 1951. 